\title{
Preservation of aberrant right hepatic arteries does not affect safety and oncological radicality of pancreaticoduodenectomy- own results and a systematic review of the literature
}

\author{
Cedrik Pyras ${ }^{1}$, Carsten Lukas ${ }^{2}$, Monika Janot-Matuschek ${ }^{1}$, Torsten Herzog ${ }^{1}$, Andrea Tannapfel ${ }^{3}$, \\ Waldemar Uhl ${ }^{1}$, Orlin Belyaev ${ }^{1}$ \\ ${ }^{1}$ Department of Surgery, St. Josef Hospital, Ruhr University Bochum, Bochum, Germany; ${ }^{2}$ Institute of Radiology, St. Josef Hospital, Ruhr University \\ Bochum, Bochum, Germany; ${ }^{3}$ Institute of Pathology, Ruhr University Bochum, Bochum, Germany \\ Contributions: (I) Conception and design: O Belyaev, C Pyras; (II) Administrative support: W Uhl, C Lukas, A Tannapfel; (III) Provision of study \\ materials or patients: W Uhl, C Lukas, A Tannapfel; (IV) Collection and assembly of data: All authors; (V) Data analysis and interpretation: C Pyras, \\ M Janot-Matuschek, T Herzog, W Uhl, O Belyaev; (VI) Manuscript writing: All authors; (VII) Final approval of manuscript: All authors. \\ Correspondence to: Orlin Belyaev, MD, PhD. Department of Surgery, St. Josef Hospital, Ruhr University Bochum, Gudrunstr. 56, 44791 Bochum, \\ Germany. Email: orlin.belyaev@klinikum-bochum.de.
}

Background: Aberrant right hepatic arteries (aRHA) are frequently encountered during pancreaticoduodenectomy (PD). Their effects on surgical morbidity and resection margin are still debated. This study aimed to compare the short term and long term outcomes in patients with and without aRHA.

Methods: A single-center retrospective analysis of 353 consecutive PD during a 5-year period was done. The type of arterial supply was determined preoperatively by CT and confirmed at surgery. Hiatt types III-VI included some type of aRHA and comprised the study group. Hiatt types I and II were considered irrelevant for PD and used as controls. Primary endpoints were the rates of major postoperative complications and the rate of R0-resection in cases of malignant disease. Secondary endpoints included duration of surgery, postoperative stay, number of harvested lymph nodes and survival in patients with pancreatic cancer. Own results were compared to existent data using a systematic review of the literature.

Results: No aRHA had to be sacrificed or reconstructed. Surgical morbidity and specific complications such as post-pancreatectomy hemorrhage (PPH), pancreatic fistula and bile leak were the same in patients with and without aRHA. There was no significant difference in operative time, blood loss, length of ICUand hospital stay. Patients with malignancy had similar high rates of R0-resection and identical number of harvested lymph nodes. Survival of patients with pancreatic cancer was not affected by aRHA.

Conclusions: aRHA may be preserved in virtually all cases of PD for resectable pancreatic head lesions without increasing surgical morbidity and without compromising oncological radicality in patients with cancer, provided the variant anatomy is being recognised on preoperative CT and a meticulous surgical technique is used.

Keywords: Replaced hepatic artery; accessory hepatic artery; pancreatic cancer; pancreatic surgery; resection margin; complications; vascular anomalies

Submitted Mar 06, 2020. Accepted for publication May 07, 2020.

doi: $10.21037 / \mathrm{hbsn}-20-352$

View this article at: http://dx.doi.org/10.21037/hbsn-20-352 


\section{Introduction}

Pancreaticoduodenectomy (PD) is one of the most complex abdominal surgical procedures. It has a wide spectrum of indications, including resectable periampullary cancer, symptomatic benign lesions of the pancreatic head as well as chronic pancreatitis. Centralisation of care and improvements in surgical technique and intensive medicine reduced mortality to less than $5 \%$ at dedicated institutions. However, postoperative morbidity still remains high, ranging between $40 \%$ and $70 \%(1,2)$. Results of surgery might be worse in patients with aberrant arterial supply to the liver, especially if not recognized promptly. Of special relevance is the most common vascular anomaly, i.e., the aberrant right hepatic artery (aRHA), which is found in $15-35 \%$ of patients undergoing $\mathrm{PD}$, which may present as a replaced vessel or as an accessory one in addition to a normal hepatic artery $(3,4)$. On the one hand a damaged or ligated aRHA may lead to impaired perfusion and ischemia of the remnant bile duct and/or parts of the liver with consecutive biliary leakage (BL) through breakdown of the hepaticojejunostomy ( $\mathrm{HJ})$, liver abscess or even hepatic failure (5). On the other hand, preservation of aRHA at the price of damage to the adventitia may lead to pseudoaneurysm formation with the risk of life-threatening delayed post-pancreatectomy hemorrhage (PPH) especially in cases of BL or postoperative pancreatic fistula (POPF) $(3,6)$. Theoretically, preservation of aRHA may also present an additional hazard to achieving a tumor-free (R0) resection margin and negatively affect the number of harvested lymph nodes in patients with malignant disease, thus putting at risk the oncological radicality of $\operatorname{PD}(7,8)$.

The aim of the present study was to investigate the incidence and management of aRHA in a large cohort of consecutive PD and to assess its effect on the surgical and oncological outcomes, and to compare the results to those already available in the literature.

\section{Methods}

\section{Patients}

This study was approved by the Ethics committee at the Ruhr University Bochum (Nr. 18-6628-BR) and done in accordance with the Helsinki Declaration as revised in 2013. Informed consent for surgery as well as for data collection and analysis was given by all patients. Between January 2014 and December 2018, 353 consecutive patients underwent primary elective open PD for benign and malignant disease.
Clinical, pathological and radiological data as well as perioperative outcomes were prospectively recorded. Based on preoperative triphasic, contrast-enhanced multidetector computed tomography (CT) using a dedicated pancreatic protocol an aRHA was reliably identified or ruled out in all patients (Figure 1). Images were saved and analyzed by a radiologist and a pancreatohepatobiliary surgeon using the house intern picture archiving and communication system (PACS). Radiological findings were confirmed at surgery. The Hiatt classification was used to divide the cohort into aRHA (Hiatt types III to VI) and control (Hiatt types I and II) groups (9).

\section{Surgical procedure}

The operations were performed by 4 experienced pancreatic surgeons according to a standardized surgical technique of dissection and reconstruction: after entering the omental bursa and mobilization of the right hemicolon, the duodenum was kocherised up to the origin of the superior mesenteric artery (SMA). Superior mesenteric vein was exposed, hepatogastric ligament opened, lymph nodes around the common hepatic artery removed and the gastroduodenal artery (GDA) identified. Suprapancreatic dissection of the hepatoduodenal ligament included isolation of the common bile duct (CBD) and identification of the portal vein above the pancreas. A standard lymphadenectomy including lymph node groups 5, 6, 8, 12-14 and 17 was performed, clearing all lymphatic tissue around the hepatic artery and the portomesenteric axis. Further lymph nodes (e.g., groups $15,16)$ were harvested only if tumor invasion was strongly suspected. Special attention was paid to avoid damage to an aRHA during dissection and division of the CBD. After being identified aRHA was carefully dissected along its entire course up to its origin and preserved (Figure 2). Reconstruction included an end-to-side, duct-to-mucosa, double-layer pancreaticojejunostomy (PJ) using interrupted polydioxanone (PDS) 5-0 suture for the outer layer and interrupted polypropylene 5-0 suture for the inner layer. Neither sealants, nor stents were applied at the PJ. A singlelayer end-to-side HJ using interrupted PDS 5-0 suture was performed. In patients with thin walled and tiny hepatic ducts, PDS 6-0 was used and the HJ was splinted using an externally diverted T-tube at discretion of the surgeon. Antecolic duodenojejunostomy was performed $50 \mathrm{~cm}$ distal to $\mathrm{HJ}$ in a double-layer continuous PDS 5-0 suture technique. In patients without pylorus preservation a Braun 


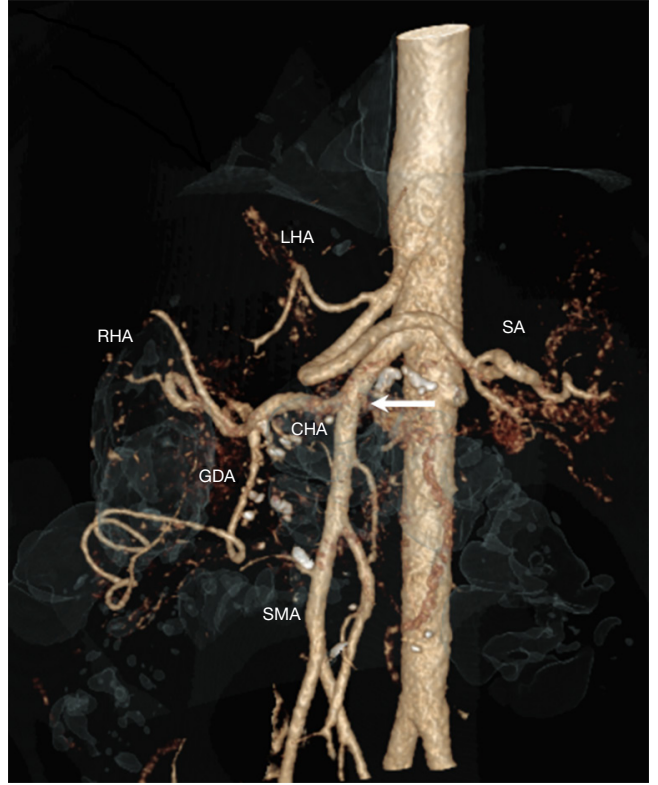

Figure $13 \mathrm{D}$-reconstruction of visceral arteries based on the arterial phase of CT showing common hepatomesenteric trunk (Hiatt type V). The arrow points at the root of CHA originating from SMA. SMA, superior mesenteric artery; CHA, common hepatic artery; RHA, right hepatic artery; LHA, left hepatic artery; SA, splenic artery; GDA, gastroduodenal artery.

enteroenterostomy was added to gastrojejunostomy. All patients received two intraabdominal soft silicone drains placed in the vicinity of the $\mathrm{HJ}$ and $\mathrm{PJ}$, derived separately through the skin of the right and left middle abdomen. Perioperative octreotide was routinely administered in all patients.

\section{Postoperative outcome}

POPF, PPH and delayed gastric emptying (DGE) were defined according to the consensus definitions by the International Study Group on Pancreatic Surgery $(6,10,11)$. All complications were classified according to the criteria of Clavien and Dindo, whereas grade III or greater were regarded as major complications (12). All deaths occurring within 30 days after surgery or throughout the hospital stay were classified as surgical mortality. Complete and detailed pathological reports were available for all patients. Patients with benign disease were excluded from the analysis of oncological results. The status of circumferential resection margin (CRM), number of harvested lymph nodes and the lymph node ratio were reported for patients with malignant

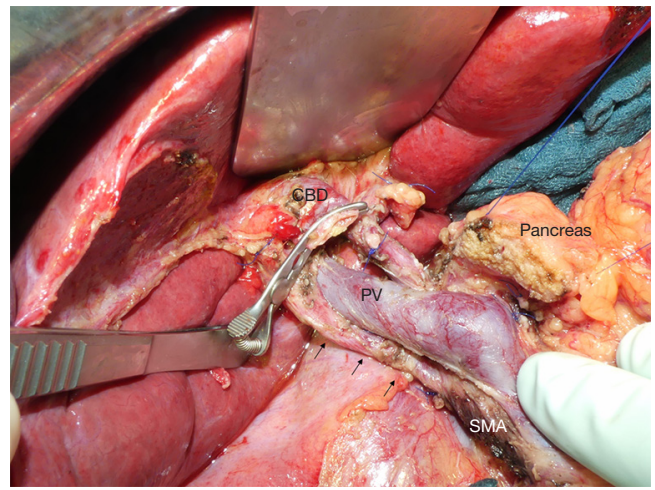

Figure 2 Intraoperative view of PD after completed resection, arrows showing the aRHA from SMA coming from beneath the PV and then dorsolateral to the CBD. CBD, common bile duct; PV, portal vein; SMA, superior mesenteric artery.

lesions. CRM-positive findings (R0-narrow) were those with vital tumor cells within $1 \mathrm{~mm}$ of resection margin. Specimen with a tumor free resection margin $>1 \mathrm{~mm}$ were reported as R0-wide. Survival analysis was performed for patients with PDAC.

\section{Systematic review of the literature}

A search with the key-words "pancreatoduodenectomy", "right hepatic artery", "hepatic artery variation", "aberrant artery" in the PubMed database was performed to find relevant publications dealing with the effects of aRHA on clinical outcome in PD for the period 1950-2019. Only comparative studies with sufficient data on postoperative course and oncological outcome were selected according to the PRISMA flow diagram (Figure 3). Pure radiological and anatomical reports without clinical data were excluded. Raw data of the included publications regarding the primary and secondary endpoints of the present study were summarized.

\section{Statistics}

Data were expressed as percentages, mean \pm standard deviation (SD) or median with minimum-maximum range. An independent samples two-tailed $t$-test (for normally distributed data) and Mann-Whitney U-test (for abnormally distributed data) were used to compare continuous variables between the study groups. The chi-squared test was used for categorical variables. Kaplan-Meier curves and $\log$ rank test were used for the survival analysis. Events were considered statistically significant if $\mathrm{P}<0.05$. The analysis 


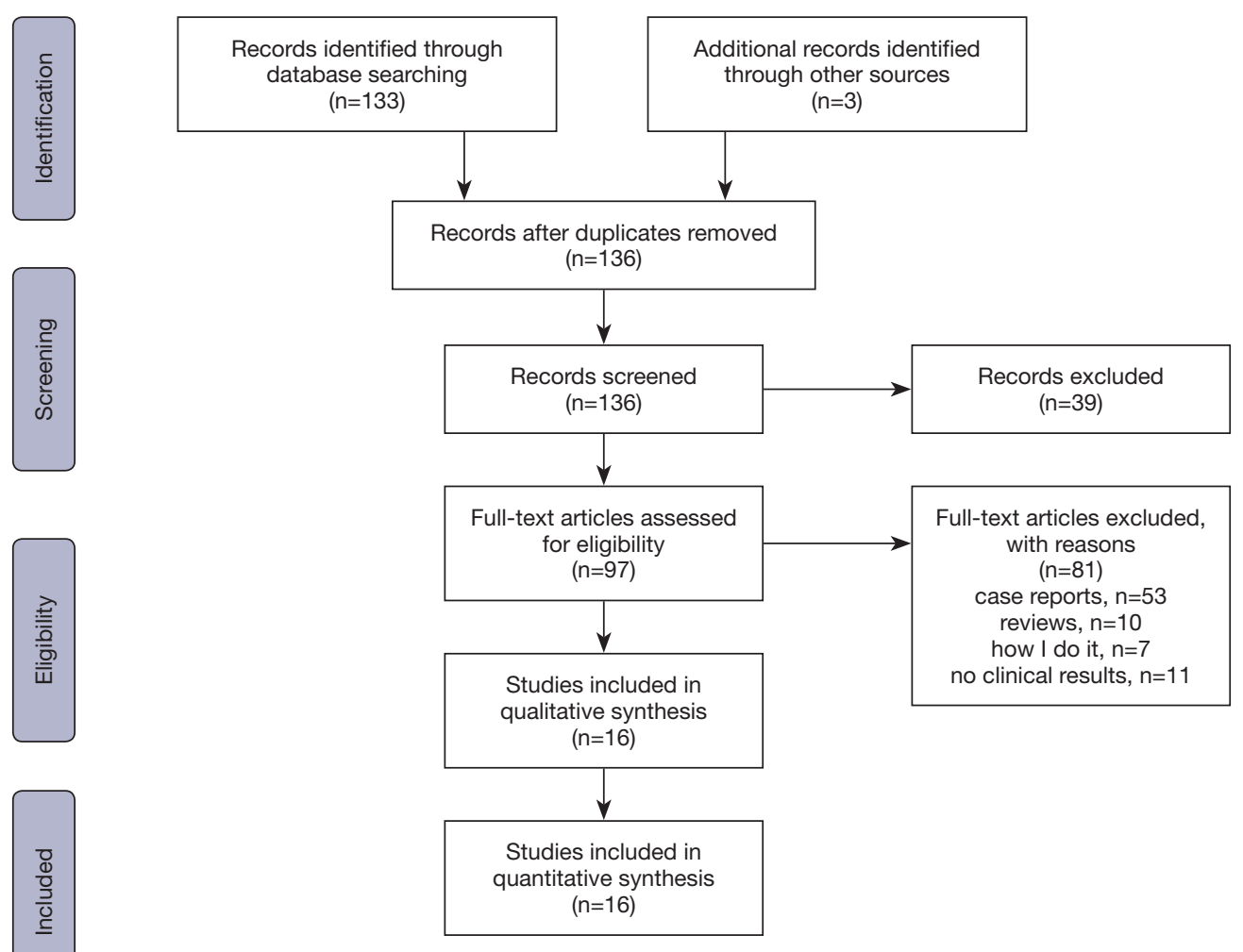

Figure 3 PRISMA flow diagram of the study.

Table 1 Distribution of study patients according to Hiatt's classification

\begin{tabular}{llc}
\hline Hiatt types & Description & Number of patients (\%) \\
\hline Type I & Standard anatomy & $240(68.0)$ \\
Type II & Replaced or accessory left hepatic artery & $33(9.3)$ \\
Type III & Replaced or accessory right hepatic artery & $56(15.9)$ \\
Type IV & Combination of type II + type III & $12(3.4)$ \\
Type V & Common hepatic artery from SMA & $11(3.1)$ \\
Type VI & Common hepatic artery from aorta & $1(0.3)$ \\
\hline
\end{tabular}

SMA, superior mesenteric artery.

was performed with SPSS software release 22.0 (SPSS Inc., Chicago, Ill., USA).

\section{Results}

\section{Comparison of short term outcome in all patients}

Eighty patients (22.7\%) had any type of aRHA (Table 1). Characteristics of the patients with aberrant and normal
RHA are summarized in Table 2. There were no significant differences in terms of age, sex, BMI, major comorbidities and ASA class between these two groups. Rates of ppPD, venous reconstructions, use of T-tubes and mode of reconstruction were identical. No arterial resection/ reconstruction was necessary in any patient. Two thirds of patients were treated for malignant disease, pancreatic ductal adenocarcinoma (PDAC) being the most common type of tumor. Patients with and those without aRHA 
Table 2 Patient characteristics

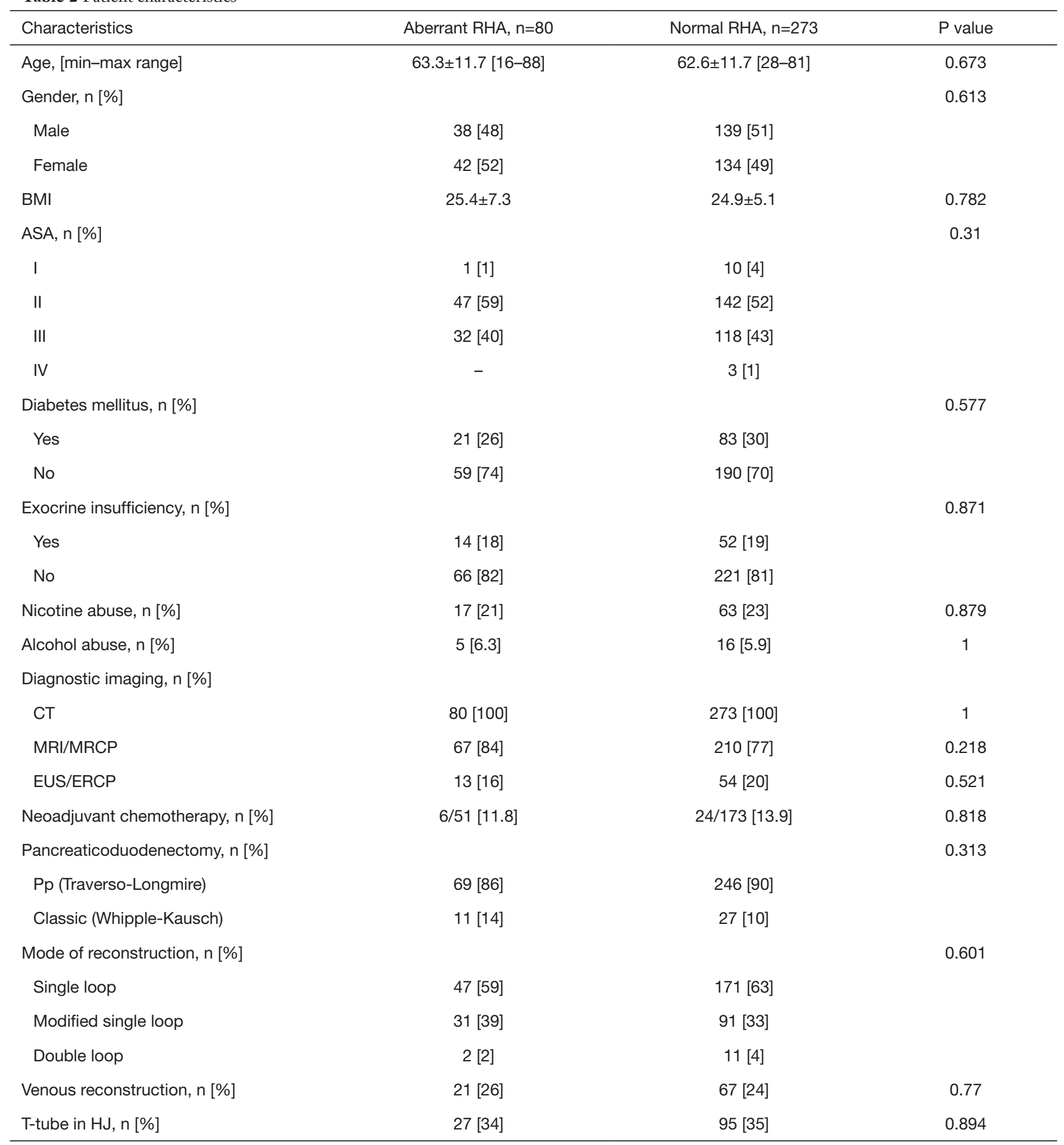

$\mathrm{RHA}$, right hepatic artery. 
Table 3 Distribution of diagnoses in 353 PD, based on final histology

\begin{tabular}{lcc}
\hline Diagnosis & Aberrant RHA, $\mathrm{n}=80$ & Normal RHA, $\mathrm{n}=273$ \\
\hline Pathology, $\mathrm{n}[\%]$ & $51[64]$ & $173[63]$ \\
Malignant & $25[31]$ & $108[40]$ \\
PDAC & $17[22]$ & $31[10]$ \\
Other pancreatic cancer & $2[2.5]$ & $10[4]$ \\
Distal bile duct cancer & $2[2.5]$ & $3[1]$ \\
Duodenal cancer & $4[5]$ & $19[7]$ \\
Ampullary cancer & $1[1]$ & $2[1]$ \\
Metastasis & $29[36]$ & $100[37]$ \\
Benign & $16[20]$ & $74[27]$ \\
Chronic pancreatitis & $11[14]$ & $15[6]$ \\
IPMN & - & $6[2]$ \\
SCN & $1[1]$ & $2[1]$ \\
SPN & $1[1]$ & $3[1]$ \\
MCN & 197
\end{tabular}

PD, pancreaticoduodenectomy; RHA, right hepatic artery; PDAC, pancreatic ductal adenocarcinoma; IPMN, intraductal papillary mucinous neoplasia; SCN, serous cystic neoplasia; SPN, solid pseudopapillary neoplasia; MCN, mucinous cystic neoplasia.

didn't differ with regards to diagnosis distribution (Table 3). Tumor size, tumor stage, lymph node infiltration, number of harvested lymph nodes and the R0-resection rate were identical for patients with cancer irrespective of the presence of aRHA (Table 4). Neither intraoperative parameter nor postoperative outcome measures differed between the groups. Operative time and blood loss were not increased in patients with aRHA. Postoperative morbidity and mortality were the same. ICU- and total postoperative stay were also not affected (Table 5). No differences were detected in the subgroup comparison of the six Hiatt types of vessel anatomy.

\section{Comparison of long-term outcome in oncologic patients}

Survival analysis was performed only for patients with PDAC because of the small numbers and heterogeneous distribution of other types of periampullary cancer in both study groups. The estimated overall median survival for 133 patients with PDAC was $29 \pm 3$ months. The mean follow-up was $25 \pm 17$ (median 21, interquartile range, 11.5-36) months. Nineteen patients were lost to follow-up after a median of 23 months. Six percent (8/133) of patients had no chemotherapy. Thirty patients $(22.5 \%)$ received neoadjuvant chemotherapy. All patients with neoadjuvant chemotherapy for an initially borderline resectable or locally advanced PDAC underwent a R0-resection and there was no difference in the oncological radicality between patients with and without aRHA in this specific subgroup. Median overall survival for patients undergoing PD for PDAC with aRHA did not differ compared to those with normal vessel anatomy: $28 \pm 4.2$ (95\% CI, 19.8-36.2) months vs. $30 \pm 4.2$ (95\% CI, 21.8-38.2) months, $\mathrm{P}=0.44$. Estimated 5-year survival rates were respectively $25 \%$ vs. 34\% in both groups-log rank test for comparison of survival distribution with continuity correction showed no difference between the groups, $\mathrm{P}=0.53$. The predominant type of recurrence was diffuse metastatic disease with the liver as the most common site. Local recurrence was diagnosed in only a small proportion of patients. Adjuvant chemotherapy was performed according to the German guidelines for treatment of pancreatic cancer with the majority of patients receiving gemcitabine chemotherapy for 6 months. mFOLFIRNOX was used in 40/133 patients and gemcitabine/nab-paclitaxel in 25 patients with PDAC as a first- or second-line chemotherapy. A total of 18 patients needed third up to fifth-line chemotherapy during followup. Radiotherapy was seldom performed (12/133) and preserved for patients with symptomatic local recurrence. 
Table 4 Oncologic results for patients with primary cancer of the pancreas and periampullary region, $\mathrm{n}=221$

\begin{tabular}{|c|c|c|c|}
\hline Variable & Aberrant RHA, $n=50$ & Normal RHA, $n=171$ & $P$ value \\
\hline T-stage, n [\%] & & & 0.51 \\
\hline T1 & $6 / 50$ [12] & 22/171 [13] & \\
\hline T2 & $11 / 50[22]$ & $43 / 171[25]$ & \\
\hline T3 & $33 / 50[66]$ & 102/171 [60] & \\
\hline T4 & - & 4 [2] & \\
\hline Tumor size $(\mathrm{cm})$ & $3.9 \pm 1.3$ & $3.82 \pm 1.2$ & 0.423 \\
\hline Harvested lymph nodes [range] & $25.3 \pm 7.9[12-44]$ & $24.4 \pm 8.4[10-61]$ & 0.482 \\
\hline Metastatic lymph nodes [range] & $2.2 \pm 2.9[0-11]$ & $2.9 \pm 3.9[0-20]$ & 0.242 \\
\hline LNR & $0.09 \pm 0.12[0-0.41]$ & $0.12 \pm 0.17[0-0.8]$ & 0.197 \\
\hline Patients with $L N R \geq 0.2, \mathrm{n}[\%]$ & 9/50 [18] & $36 / 171$ [21] & 0.695 \\
\hline Resection margin, $n$ [\%] & & & 0.503 \\
\hline R0 wide & 36 [71] & $112[65]$ & \\
\hline R0 narrow & $9[17]$ & 44 [25] & \\
\hline R1 & $6[12]$ & $17[10]$ & \\
\hline Median survival (months) & $28 \pm 4.2$ & $30 \pm 4.2$ & 0.44 \\
\hline Estimated 5-year survival rate & $25 \%$ & $34 \%$ & 0.532 \\
\hline Local recurrence PDAC, n [\%] & $3 / 25$ [12] & 18/108 [16] & 0.764 \\
\hline Metastasis PDAC, n [\%] & $16 / 25[64]$ & $61 / 108[56]$ & 0.654 \\
\hline
\end{tabular}

RHA, right hepatic artery; LNR, lymph node ratio; PDAC, pancreatic ductal adenocarcinoma.

\section{Systematic review of the literature}

There were 16 retrospective studies comparing the clinical outcome of 3,203 patients with and without aRHA. Table 6 represents a summary of the major surgical outcomes of these studies including our own data. aRHA didn't correlate to increased mortality and morbidity in any of the studies. The lymph node yield, the rate of tumor-free resection margin and long-term survival were also not affected by the presence of aRHA in oncologic patients. Six studies found significantly longer duration of surgery in patients with aRHA $(7,8,13-26)$.

\section{Discussion}

Anatomic variations of the hepatic artery, first described by Haller in 1756 , have drawn the increasing interest of the surgical community with the rise of complex hepatobiliary and pancreatic resections in the second half of the twentieth century (27). The first useful and generally applicable classification was based on 200 autopsies and published by Michels in 1966 (28). Some thirty years later, Hiatt et al. proposed a similar classification based on 1000 angiographies, which was in fact a simplified modification of the Michels system, omitting the differentiation between replaced and accessory right hepatic arteries (9). Despite numerous further attempts of improve, the classification system of Hiatt still remains the most widely accepted due to its simplicity and practicability. According to Hiatt et al. an aberrant right hepatic artery of any type can be found in one of four patients (24\%) and similar results ranging between $15 \%$ and $35 \%$ have been confirmed by most authors $(3,14,16)$. In our cohort of 353 consecutive patients the rate of aRHA was almost $23 \%$, being very close to that reported by Hiatt. This makes aRHA the most frequently encountered and thus the most relevant vascular anomaly during PD. An aRHA, being a completely replaced or just an accessory one, may arise from the SMA or directly the aorta, passing on the back surface or right through the head of the pancreas and behind the portal vein to join the hepatoduodenal ligament on the dorsolateral 
Table 5 Summary of results from 16 retrospective studies and own data on the impact of aRHA on PD

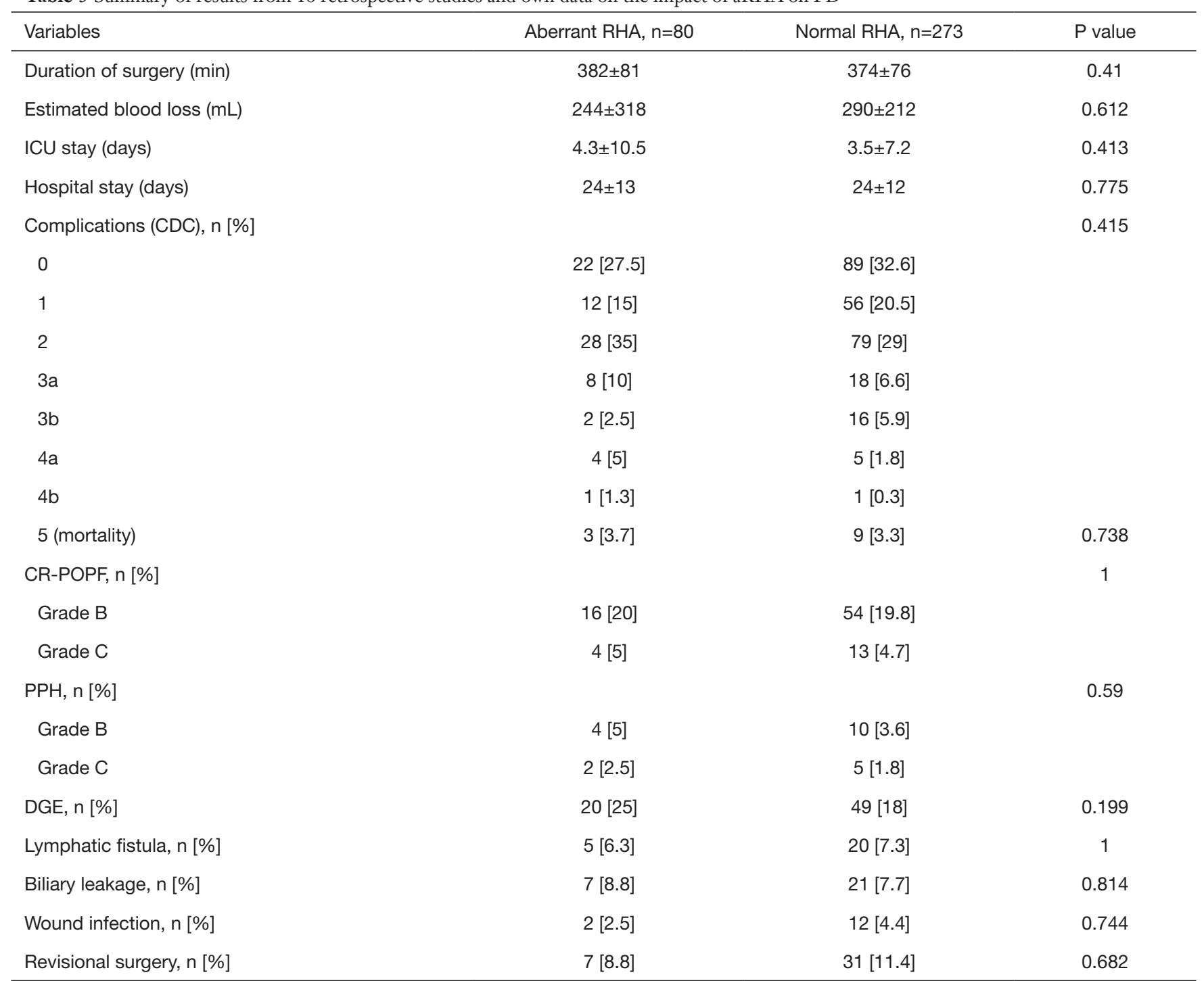

$\mathrm{PD}$, pancreaticoduodenectomy; aRHA, aberrant right hepatic artery; CDC, Clavien-Dindo Classification; CR-POPF, clinically relevant postoperative pancreatic fistula; $\mathrm{PPH}$, postpancreatectomy hemorrhage; DGE, delayed gastric emptying

side. Therefore, aRHA is susceptible to abutment by large benign lesions or infiltration by periampullary cancer and may be encased by inflammatory tissue in severe chronic pancreatitis. The risks of intraoperative vascular injury, blood loss, prolonged operative time, and postoperative morbidity might increase. The increased risk for positive resection margins and lower lymph node yield might theoretically have a negative effect on long term survival of oncologic patients with aRHA undergoing PD.

The most important first step in preventing such risks is the early and reliable identification of vessel variations and their exact relationship with neighbour structures. Our results show that aRHA can preoperatively be detected in $100 \%$ of studied patients using a high-quality, thin-sliced, contrast enhanced CT with an arterial phase. Modern software allows multiplanar 3D-reconstructions within seconds. Nevertheless, we use seldom such $3 \mathrm{D}$-images, since identification of vessels is usually straightforward using the standard axial and coronal planes. None of the patients in this study received preoperative angiography. The CT findings were confirmed at surgery in all patients. The high diagnostic accuracy of CT was confirmed in the study of 


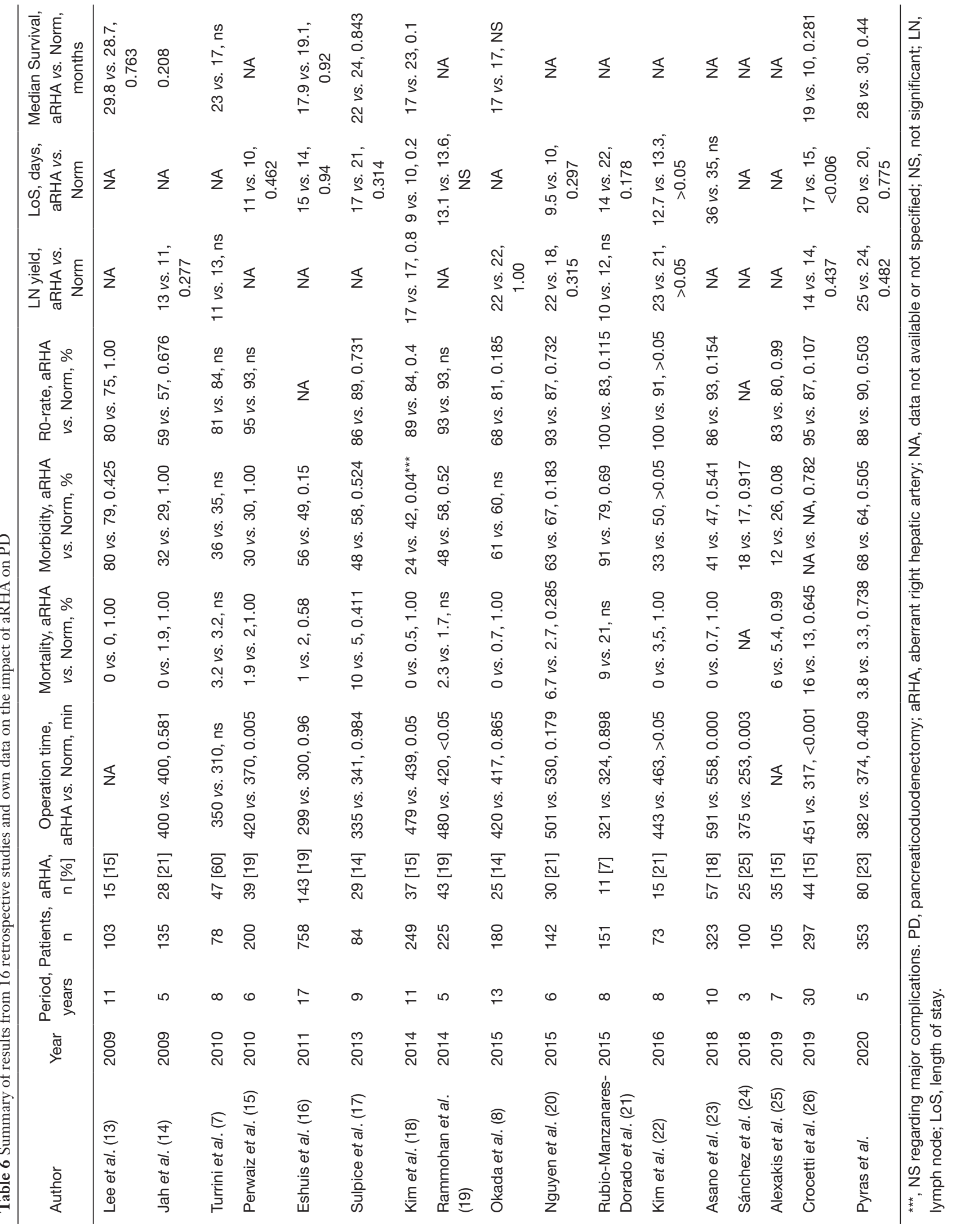


Balzan et al., and besides meticulous surgical technique, may partially explain the successful preservation of all aRHA in our cohort (29). Considering this background, we do not have any explanation for some very low rates of aRHA recognition using CT as low as $50-70 \%$, recently reported $(19,22,24)$.

Duration of surgery and blood loss were not increased in patients with aRHA in our study, corresponding to the majority of other reports. This is mostly due to the preservation of aRHA. Indeed, the few studies with significantly longer operation times and increased blood loss were at the same time those with the highest rates of arterial reconstructions. For example, Asano et al. had to resect aRHA in $18 \%$ of the patients with this anomaly and reported a prolongation of surgery of about 33 minutes (23). Crocetti et al. reconstructed $25 \%$ of aRHA, which prolonged operating time with 34 minutes (26). Rammohan et al. ligated aRHA in $19 \%$ and reconstructed it in additional $2 \%$ resulting in a 60 minutes difference in the operating time (19). Nevertheless, neither prolonged surgery, nor increased blood loss affected morbidity and mortality. These outcomes were irrespective of the presence of aRHA in all 16 published studies and confirmed by our own results. Respectively, ICU- and postoperative hospital stay were also not affected by the presence of aRHA.

Our study showed that oncological radicality of PD is not necessarily compromised by the preservation of aRHA. There was only one other study by Lee $e t$ al. with $100 \%$ preservation of aRHA (13). In all other studies preservation rate ranged between $76 \%$ and $94 \%$. However, these results are difficult to compare, since raw data on selection criteria for surgery at the different institutions are missing and surgical strategies in the presence of arterial involvement may vary. Our strategy for patients with PDAC since 2013 is based on a very liberal indication for neoadjuvant chemotherapy for all patients with LAPC/ BRPC due to suspicion of tumor infiltration of the hepatic arteries, as provided by high-quality preoperative CT scans. Consequently, only clearly resectable lesions with definitively free hepatic vessels are scheduled for PD. In the rare cases of unexpected real tumor involvement of the hepatic vessels we do perform arterial resection and reconstruction using the stump of GDA or a splenic artery switch, or a prosthesis on the very rare occasion, combined with a completion pancreatectomy to avoid PPH due to a POPF. This strategy may explain the $100 \%$ preservation of aRHA in our cohort of patients. This means by no way that only tumours of an early stage were scheduled for surgery- as a matter of fact two thirds of our patients suffered a T3lesion, $20 \%$ had a lymph node ratio $>0.2,25 \%$ needed some type of venous reconstruction, and 30 patients had neoadjuvant chemotherapy for initial BRPC/LAPC.

The excellent oncologic radicality of $\mathrm{PD}$ in patients with malignant disease with $90 \%$ tumor free margin and the highest average number of harvested lymph nodes per patient compared to all other studies, despite aRHA preservation in $100 \%$ of cases, remains the most important finding of this study and makes the difference to other recent publications. The study by Crocetti et al. is a fine example of the effects of aggressive strategy with high resection rates in cases of aRHA: in their study a resection of aRHA with reconstruction was performed in $25 \%$, which yielded a very high R0 rate of up to $95 \%$ (26). However, the price for this aggressive strategy was high: a mortality of $16 \%$, prolonged operative time by more than 2 hours, increased blood loss, prolonged ICU and total hospital stay. Considering our results, this means that a resection of aRHA during PD for PDAC compared to its preservation would mean a gain of $5 \%$ in $\mathrm{R} 0$ resection rate $(95 \%$ vs. $90 \%)$ at the price of almost $500 \%$ increased risk or mortality (16\% vs. $3.5 \%)$.

The study of Asano et al. shows further that a macroscopically suspected tumor infiltration of aRHA does not necessarily mean a real one: they resected aRHA in 9/51 patients in order to achieve R0 margin, however real tumor infiltration of aRHA was confirmed in none of the patients at final pathology. Moreover, despite of aRHA resection, R0 was achieved in only 6/9 patients because of tumor residual at other places such as pancreatic remnant and retropancreatic margin (23). Similar results were reported by Okada, who resected 9/25 aRHA to achieve tumor free resection margin, however accomplished a tumor free margin in only 2 of 9 patients (8). Thus, our study results cannot confirm the statement of Okada, that aRHA should generally be divided to achieve R0 resection. Asano showed however, that resection of replaced RHA without reconstruction has no negative effects on morbidity with only one case of postoperative hepatic abscess (23). This may be explained by the presence of hilar plate collaterals from the left hepatic artery, as well as additional hepatic vascularization by the inferior phrenic artery and the right adrenal artery. As a proof of that, the authors observed a significant backflow in all cases of transected aRHA and proved the intact blood flow by intraoperative Doppler. The statement by Asano is confirmed by the results of Okada, who reported a preoperative coil embolization of aRHA in 


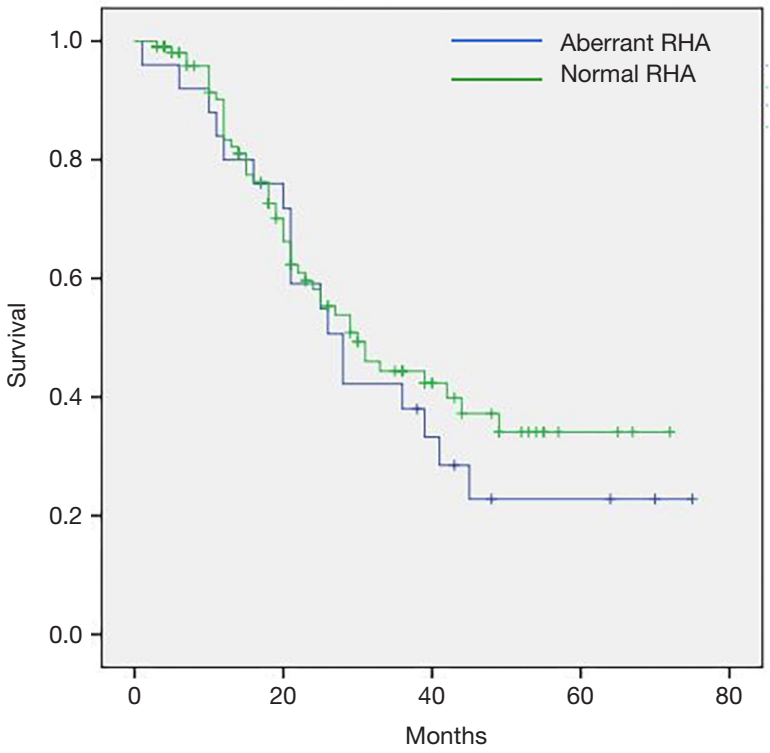

Figure 4 Kaplan-Meier survival curves for patients with PDAC. RHA, right hepatic artery; PDAC, pancreatic ductal adenocarcinoma.

6 patients, who underwent then a resection of aRHA during PD and none of them suffered postoperative liver morbidity $(8,23)$.

Strengths of the present study include the large cohort of consecutive patients treated by a small group of experienced surgeons according to a standard surgical technique and being managed following established perioperative protocol over a relatively short period of time. This standardized management at a single center allows better reproducibility and internal validity of results. This study represents the second largest single center experience in PD with aRHA. Eshuis et al. reported a larger cohort, however over a much longer study period of 17 years (16). Resection of hepatic arteries, especially the CHA due to tumor invasion should be critically discussed in the era of powerful chemotherapeutic regimes for neoadjuvant treatment. According to the internationally accepted NCCN criteria a tumor invasion of the hepatic artery determines PDAC as locally advanced - primary resection of such LAPC is not indicated, since despite its technical feasibility it cannot offer a survival benefit and increases morbidity and mortality dramatically $(30,31)$.

Regarding long term survival, the studies by Eshuis et al., Crocetti et al., and Kim et al. already proved that survival was not affected by aRHA $(16,18,26)$. Our results support these data and reveal the longest median survival of 29 months among all studies included in the systematic review (Figure 4). Similar survival times were reported only by Lee et al., however all patients included in their study suffered bile duct cancer and our survival data refer only to patients with PDAC (13).

Limitations of the study are its retrospective character and the lack of comparison to patients with sacrificed or reconstructed aRHA. Indeed, we did not have any case of planned PD during this study period of 5 years with a sacrificed or reconstructed normal or aberrant RHA. In our experience these measures were always a part of emergency revisional surgery for bleeding complications, when angiography failed to solve the problem. In such instances we prefer to sacrifice the right hepatic artery rather than reconstruct it. In our opinion, this strategy is easier and faster than arterial reconstruction and usually does not lead to life-threatening complications itself. Further limitation may be the fact that our results apply only to open PD, so we cannot automatically transfer our conclusions to minimally invasive PD. However, there are already two studies, which showed that robotic PD is feasible in the presence of aRHA in a selected group of patients $(20,22)$.

In summary, the results of the present study and the systematic review of existent literature clearly confirm that the presence of aRHA does not affect negatively the short-term and long-term clinical outcome of PD. Reliable identification of aRHA on contrast enhanced CT combined with standardized meticulous surgical technique allow its safe preservation without compromising oncological radicality of the procedure in patients with pancreatic cancer.

\section{Acknowledgments}

Funding: None.

\section{Footnote}

Conflicts of Interest: All authors have completed the ICMJE uniform disclosure form (available at https://hbsn. amegroups.com/article/view/10.21037/hbsn-20-352/coif). The authors have no conflicts of interest to declare.

Ethical Statement: The authors are accountable for all aspects of the work in ensuring that questions related to the accuracy or integrity of any part of the work are appropriately investigated and resolved. This study was approved by the Ethics committee at the Ruhr University 
Bochum (Nr. 18-6628-BR) and done in accordance with the Helsinki Declaration as revised in 2013. Informed consent for surgery as well as for data collection and analysis was given by all patients.

Open Access Statement: This is an Open Access article distributed in accordance with the Creative Commons Attribution-NonCommercial-NoDerivs 4.0 International License (CC BY-NC-ND 4.0), which permits the noncommercial replication and distribution of the article with the strict proviso that no changes or edits are made and the original work is properly cited (including links to both the formal publication through the relevant DOI and the license). See: https://creativecommons.org/licenses/by-nc-nd/4.0/.

\section{References}

1. Cameron JL, He J. Two thousand consecutive pancreaticoduodenectomies. J Am Coll Surg 2015;220:530-6.

2. Keck T, Wellner UF, Bahra M, et al. Pancreatogastrostomy versus Pancreatojejunostomy for RECOnstruction after PANCreatoduodenectomy (RECOPANC, DRKS 00000767). Perioperative and long-term results of a multicenter randomized controlled trial. Ann Surg 2016;263:440-9.

3. El Amrani M, Pruvot FR, Truant S. Management of the right hepatic artery in pancreaticoduodenectomy: a systematic review. J Gastrointest Oncol 2016;7:298-305.

4. Shukla PJ, Barreto SG, Kulkarni A, et al. Vascular anomalies encountered during pancreatoduodenectomy: do they influence outcomes? Ann Surg Oncol 2010;17:186-193.

5. Traverso LW, Freeny PC. Pancreaticoduodenectomy. The importance of preserving hepatic blood flow to prevent biliary fistula. Am Surg. 1989;55:421-6.

6. Wente MN, Veit JA, Bassi C, et al. Post-pancreatectomy haemorrhage (PPH): an International Study Group of Pancreatic Surgery (ISGPS) definition. Surgery 2007;142:20-5.

7. Turrini O, Wiebke EA, Delpero JR, et al. Preservation of replaced or accessory right hepatic artery during pancreaticoduodenectomy for adenocarcinoma: impact on margin status and survival. J Gastrointest Surg 2010;14:1813-9.

8. Okada K, Kawai M, Hirono S, et al. A replaced right hepatic artery adjacent to pancreatic carcinoma should be divided to obtain $\mathrm{R} 0$ resection in pancreaticoduodenectomy. Langenbecks Arch Surg 2015;400:57-65.

9. Hiatt JR, Gabbay J, Busuttil RW. Surgical anatomy of the hepatic arteries in 1000 cases. Ann Surg 1994;220:50-2.

10. Bassi C, Marchegiani G, Dervenis C, et al. The 2016 update of the International Study Group (ISGPS) definition and grading of postoperative pancreatic fistula: 11 Years After. Surgery 2017;161:584-91.

11. Wente MN, Bassi C, Dervenis C, et al. Delayed gastric emptying (DGE) after pancreatic surgery: a suggested definition by the International Study Group of Pancreatic Surgery (ISGPS). Surgery 2007;142:761-8.

12. Dindo D, Demartines N, Clavien PA. Classification of surgical complications: a new proposal with evaluation in a cohort of 6336 patients and results of a survey. Ann Surg 2004;240:205-13.

13. Lee JM, Lee YJ, Kim CW, et al. Clinical implications of an aberrant right hepatic artery in patients undergoing pancreaticoduodenectomy. World J Surg 2009;33:1727-32.

14. Jah A, Jamieson N, Huguet E, et al. The implications of the presence of an aberrant right hepatic artery in patients undergoing a pancreaticoduodenectomy. Surg Today 2009;39:669-74.

15. Perwaiz A, Singh A, Singh T, et al. Incidence and management of arterial anomalies in patients undergoing pancreaticoduodenectomy. JOP 2010;11:25-30.

16. Eshuis WJ, Olde Loohuis KM, Busch OR, et al. Influence of aberrant right hepatic artery on perioperative course and longterm survival after pancreatoduodenectomy. HPB (Oxford) 2011;13:161-7.

17. Sulpice L, Rayar M, Paquet C, et al. Does an aberrant right hepatic artery really influence the short- and longterm results of a pancreaticoduodenectomy for malignant disease? A matched case-controlled study. J Surg Res 2013;185:620-5.

18. Kim PT, Temple S, Atenafu EG, et al. Aberrant right hepatic artery in pancreaticoduodenectomy for adenocarcinoma: impact on resectability and postoperative outcomes. HPB (Oxford) 2014;16:204-11.

19. Rammohan A, Palaniappan R, Pitchaimuthu A, et al. Implications of the presence of an aberrant right hepatic artery in patients undergoing pancreaticoduodenectomy. World J Gastrointest Surg 2014;6:9-13.

20. Nguyen TK, Zenati MS, Boone BA, et al. Robotic pancreaticoduodenectomy in the presence of aberrant or anomalous hepatic arterial anatomy: safety and oncologic outcomes. HPB (Oxford) 2015;17:594-9.

21. Rubio-Manzanares-Dorado M, Marín-Gómez LM, 
Aparicio-Sánchez D, et al. Implication of the presence of a variant hepatic artery during the Whipple procedure. Rev Esp Enferm Dig 2015;107:417-22.

22. Kim JH, Gonzalez-Heredia R, Daskalaki D, et al. Totally replaced right hepatic artery in pancreaticoduodenectomy: is this anatomical condition a contraindication to minimally invasive surgery? HPB (Oxford) 2016;18:580-5.

23. Asano T, Nakamura T, Noji T, et al. Outcome of concomitant resection of the replaced right hepatic artery in pancreaticoduodenectomy without reconstruction. Langenbecks Arch Surg 2018;403:195-202.

24. Sánchez AM, Tortorelli AP, Caprino P, et al. Incidence and Impact of Variant Celiacomesenteric Vascularization and Vascular Stenosis on Pancreatic Surgery Outcomes: Personal Experience. Am Surg 2018;84:181-7.

25. Alexakis N, Bramis K, Toutouzas K, et al. Variant hepatic arterial anatomy encountered during pancreatoduodenectomy does not influence postoperative outcomes or resection margin status: A matched pair analysis of 105 patients. J Surg Oncol 2019;119:1122-7.

Cite this article as: Pyras C, Lukas C, Janot-Matuschek M, Herzog T, Tannapfel A, Uhl W, Belyaev O. Preservation of aberrant right hepatic arteries does not affect safety and oncological radicality of pancreaticoduodenectomy-own results and a systematic review of the literature. HepatoBiliary Surg Nutr 2022;11(1):25-37. doi: 10.21037/hbsn-20-352
26. Crocetti D, Sapienza P, Ossola P, et al. Does Aberrant Right Hepatic Artery Influence the Surgical Short- and Long-term Outcome of Pancreatoduodenectomy? In Vivo 2019;33:1285-92.

27. Haller A, Rollinus CJ. Icones anatomicae quibus praecipuae partes corporis humani exquisita cura delineatae continentur. Gottingen, 1756.

28. Michels NA. Newer anatomy of the liver and its variant blood supply and collateral circulation. Am J Surg 1966;112:337-47.

29. Balzan SMP, Gava VG, Pedrotti S, et al. Prevalence of hepatic arterial variations with implications in pancreatoduodenectomy. ABCD Arq Bras Cir Dig 2019;32:e1455.

30. Mollberg N, Rahbari NN, Koch M, et al. Arterial resection during pancreatectomy for pancreatic cancer: a systematic review and meta-analysis. Ann Surg 2011;254:882-93.

31. National Comprehensive Cancer Network (NCCN) Guidelines. Pancreatic Adenocarcinoma, Version 1. 2019 November 8th, 2018. 